\author{
Andrea Miranda Meza \\ Doctora en Ciencias Sociales \\ Universidad de Deusto \\ andreamirandameza@gmail.com
}

\title{
Con cuerpo y sin cuerpo: Naturaleza/ Tecnología y un nudo entre ambos
}

\section{Full-bodied and disembodied: Nature/Technology and knot between the two}

\begin{abstract}
Resumen
El cuerpo implica una alteridad, un lazo social que permite la construcción subjetiva. La tecnología le permite hacerse un cuerpo propio, construir un nuevo cuerpo de diferentes maneras. Las tecnologías en el cuerpo, la salud, las maneras de alimentarse y de habitar un espacio, esta intervención no es sólo en cada cuerpo individual sino también al conjunto del cuerpo social. El espacio entero de las existencias se politiza sobre el cuerpo.

La evolución humana podrá depender de la tecnología ¿Qué somos o estamos dejando de ser? Muchas de las preguntas dominan el paisaje de las respuestas especulativas en las que, el cuerpo, constituye el punto central. En este contexto, el cuerpo adquiere una importancia en la crítica social y la tecnología, nos permite conocer mejor las estructuras mediante el cual, el cuerpo como un conjunto de instalaciones, revelan el mapa de lo corporal en el momento presente.
\end{abstract}

Palabras clave: Cuerpo, Tecnología, Subjetividad.

\begin{abstract}
The body implies an alterity to which to offer it so that a social bond can be established allowing subjective construction. Technology allows you to make your own body, to build a new body in different ways.

Technologies, in the body, in health, in the ways of eating and inhabiting a space. The intervention is not only in each individual body but also in the whole of the social body. The entire space of stocks is politicized over the body.

Human evolution may depend on technology? What are we or are we ceasing to be? Many of the questions dominate the landscape of speculative answers in which the body is the focal point. In this context, the body becomes important in social criticism and technology allows us to better understand the structures through which the body as a set of facilities reveals the map of the body at the present moment.
\end{abstract}

Keywords: Body, technology, Subjectivity 
Andrea Miranda

\section{El Cuerpo es en el Mundo}

En ciertas tradiciones teóricas filosóficas o de la teoría social vemos como elementos fundamentales de la construcción del sujeto moderno el estudio sobre el cuerpo y la tecnología. En este proceso de construcción intersubjetiva, sobresale la idea de analizar lo que significa ser humano y cómo nuestra sociedad depende cada vez más de las infraestructuras de la comunicación como proceso de complementariedad para afrontar el mundo en el que vivimos. En el presente, nuestros propios cuerpos confinados al estudio o trabajo desde casa, apropiándonos del recurso técnico como una doble piel que actúa como mediadora entre nosotros y el mundo.

En el ámbito de la filosofía es siempre difícil, y hasta imposible, decir la "última palabra" sobre un concepto o tema, sin embargo, creemos que toda complejidad respecto al conocimiento y los asuntos humanos se ha iniciado desde mucho antes a través del cuerpo. Por tal motivo, en el presente artículo se formulan solamente posibilidades, nunca certezas.

La contemporaneidad lo exige, pareciera que el cuerpo siempre ha constituido una limitación en tanto ha exigido el hecho de su transcendencia, las que provocan nuevas realidades y formas de sentido. Las nuevas tecnologías logran borrar las fronteras espaciales y temporales, instaurando un nuevo modelo de sociedad que de alguna manera u otra nos permiten hacernos cuerpos. De tal manera la tecnología se ubica inmersa en una fase fundamental de la construcción del sujeto, en su ámbito y en su ser, que magnifica sus sensaciones, que actúa como mediador de él y del mundo, extendiendo sus sentidos, su cuerpo, sometiendo al sujeto a la ideología de lo sensible de una sensocracia tecnológica, es decir que obedece a una matriz sensorial de la lógica técnica. (Abril, 2010; 114). Como lo plantea Merleau-Ponty:

"Es en el cuerpo que se encarna el ser-en-el-mundo sin él no existiría como espacio concreto en el que se manifiesta este imaginario instituido y en el que se logra la sensocracia tecnológica, es el cuerpo, aquel que está expuesto y afectado por el tiempo, a través del cual nos ponemos en contacto con el otro, pero también el cuerpo entendido como objeto modelador de las fuerzas sociales, por lo que existe un cuerpo social y un cuerpo físico, que están en constante relación. El cuerpo entendido no sólo 
como lugar limitado en el cual llegamos a experimentar el mundo, sino a través del cual llegamos a ser vistos en él.”(Merleau-Ponty, 1996:417)

Transcurre una forma de pensar y de ser que se relaciona con el logos de la modernidad.

Hay bastantes disputas acerca de la función de la filosofía de Nietzsche a la biología. Lo que aparece más allá de esto es, sin embargo, el criticismo del racionalismo socrático como perspectiva de (forma de vida) que oscureció la importancia de la emoción y el sentimiento en la percepción humana de la realidad. (Turner, 2009; 20-21).

Para establecer esta emotividad, Nietzsche atribuyó singular importancia a la creatividad artística como actividad simultáneamente política. Apareció una visión positiva del mundo para erigir la afirmación de la vida en el cuerpo. En la filosofía de Nietzsche notamos que el cuerpo es crucial para entender los dilemas de la modernidad. El intento de Nietzsche de implicar al cuerpo en el debate de la estética fue una crítica bastante compleja. Si seguimos este recorrido vemos cómo Nietzsche vio la relación entre cultura y naturaleza en términos de dialéctica:

“Cada época en la historia de la evolución humana, por la que el Hombre transforma la naturaleza en tecnología, es también un período en el que la naturaleza del Hombre se transforma. Entonces cada período da lugar a un ideal (físico) del Hombre, con un carácter especial que es también y simultáneamente un nuevo cuerpo". (Nietzsche, 2000; 52)

Por su parte, Adorno y Horkheimer en la Dialéctica de la Ilustración reconocen una afirmación muy clara de este principio:

"Europa tiene dos historias: una historia conocida, escrita, y una historia subterránea. Esta última se basa en el hecho de que los instintos humanos y las pasiones que están desplazadas y destruidas por la civilización (...) La relación con el cuerpo humano está estropeada desde el principio". (Adorno y Horkheimer: 1994; 231) 
Los autores continúan sosteniendo que la cristiandad y el capitalismo han unido sus fuerzas para declarar que el trabajo es virtuoso, pero el cuerpo es la carne, fuente de toda maldad. La relación amor-odio con el cuerpo domina la cultura moderna. Este punto de vista crítico del cuerpo en relación con las demandas del capitalismo fue, por consiguiente, desarrollado y elaborado por Herbert Marcuse, por ejemplo, en Eros y la Civilización (1955) Marcuse sostuvo que; "mientras en las sociedades simples sería necesario un cierto grado de represión sexual para asegurar los requerimientos mínimos de la reproducción económica, el capitalismo ha producido unos excedentes económicos altos, basado en su supremacía tecnológica.” (Marcuse, 2003; 177).

Por otra parte, el análisis de Foucault de la sexualidad en Historia de la Sexualidad Volumen II (1983) dependía claramente de esta reevaluación:

“el cuerpo como banco de deseo, irracionalidad, emotividad y pasión sexual surgió, y de modo especial en la teoría social francesa, como tópico central en el escrito oposicional, como símbolo de protesta contra la racionalidad capitalista y la regulación burócrata.” Foucault, 2005; 163)

Vemos como la corporeidad es la forma de existir en el mundo, de tal modo que nuestro propio cuerpo nunca puede constituirse más que de forma sesgada y parcial, en objeto de nuestra percepción. Hay al respecto una distinción clara y tajante entre el cuerpo propio y el cuerpo objeto. La división de cuerpo y alma, como la de intelecto y sentimiento, responde a una concepción del ser humano según una filosofía mecanicista y es necesaria una concepción filosófica dialéctica en que el enfoque central sea la vida, la vida humana y sus expresiones; sería una filosofía vitalista, según dicen unos, o humanista, según dicen otros. Merleau-Ponty sostiene que; "A nivel de la percepción, no hay distinción sujeto-objeto, simplemente somos en el mundo. Quien percibe es un sujeto hecho carne con el mundo, un ser-en-el-mundo. (Merleau-Ponty, 2010; 109)

El mundo que habitamos y que somos tiene que ver con la mediación de los cuerpos de los otros con los otros. (Miñambres (Comp), 2002; 75-79). Y así, cada uno de nosotros responde a esos cuerpos, el mundo que hace que se constituya -físico y social- se convierte, así, en símbolo que se ofrece ante los demás por medio del cuerpo. 
Las relaciones que se establecen entre los seres humanos dependen del hecho de nuestra corporalidad, es decir, los significados están corporeizados y el mismo cuerpo es significado que se hace presente, como la representación del mundo, no podemos entender el cuerpo y el mundo como realidades aparte, distintas, ni tampoco reducir el uno al otro: el cuerpo comunica, hace presente su existencia, es decir, su forma de ser mundo. La metáfora, sin la que no podríamos pensar, permite al espíritu, nos dice Hannah Arendt:

"Estar vinculado constantemente al mundo de nuestra experiencia corporal y cotidiana. No hay dos mundos: uno sensible y otro no sensorial, porque la metáfora nos permite unirlos, es decir, "transferir" transformar formar transportar, meta-pherein- nuestras experiencias sensoriales para convertirse en la carne necesaria del ejercicio de nuestro pensamiento más abstracto. Los límites del cuerpo dibujan a su escala el orden moral y significativo del mundo. Pensar el cuerpo, es otra forma de pensar el mundo y el vínculo social. Un trastorno introducido en la configuración del cuerpo es un desorden introducido en la coherencia del mundo.” (Arendt, 1993; 198-202).

Los significados se producen en nuestro cuerpo, gracias a su interrelación con el medio, interrelación que convierte a este mundo en la medida en que nuestros cuerpos tienen una estructura bio-genética propia de la especie y gracias a las similitudes relativas al medio físico en que se desarrolla la vida de los seres humanos. El cuerpo, no es un objeto más, ni un producto natural sólo explicable por las leyes de la biología. Mi cuerpo, el cuerpo de cada uno de nosotros, es una construcción social que se ha ido formando en contacto con otros cuerpos, que son a su vez, lenguaje y fuente de significación. El cuerpo manifiesta su potencia transformadora del mundo y de sí mismo. En el cuerpo residiría una voluntad de poder que se ejerce desde el cuerpo y nos mueve a actuar sobre el mundo, cuya fuerza fundamental es la creación. "El poder-saber toma a su cargo la vida y eso le da acceso al cuerpo." (Foucault 2003: 88). Eso le convierte en un agente de modelación y transformación de la vida humana. El cuerpo es tema central tanto 
para develar las condiciones de posibilidad de nuestro saber actual como para indagar en las diversas formas como devenimos sujetos.

\section{El cuerpo se confunde con el Mundo}

La incapacidad estructural del cuerpo humano ya fue planteada por Bataille a principios del siglo pasado en El Ojo Pineal (1930) sin que en tales razonamientos predominara la posibilidad de la tecnología. En su lugar, el cuerpo mejorado que Bataille proponía apelaba a la parte anatómica del humano que, a lo largo de los siglos sirvió de especulación como sede de la interacción del cuerpo y el alma en Descartes, y previamente en los griegos Herófilo y Acmelón, y que durante cierto tiempo permaneció en la sombra de la anatomía y fisiología humana al ser considerado un apéndice molesto cuyas funciones no acababan de ser desveladas. (Aguilar, 2008; 128). Esta parte mítica del cuerpo servía de propósito al autor para denunciar la insuficiencia estructural del cuerpo humano. Al decir de Bataille:

"En la parte superior de nuestro cráneo se encuentra una glándula llamada, el ojo pineal. Este gran ojo sería la expresión volcánica del hombre hacia arriba, este sería el ojo con que el hombre vería al sol de frente, demostrando así la identidad con él. "El ojo se abre y con él se abre el grito que se traga a sí mismo, el grito del ojo, es el grito desesperado del "ojo" de la conciencia. En la abertura el hombre se transparenta en sus formas más degradantes, las más humanas y es a través de ellas que él habla." (Bataille, 1996; 17-21).

Cuando hacemos alusión al cuerpo, lo hacemos como si fuera una circunstancia inmediata a nuestro yo más íntimo. La incertidumbre nos ubica en la ambigüedad ¿tenemos un cuerpo o él nos tiene? La carnalidad nos confronta con nuestra infinitud por aquello del temor a desvanecerse. Los ejemplos son interminables, pero en todos ellos es posible apreciar la dicotomía como eje central del debate: natural/artificial, orgánico/inorgánico, ficción/realidad, mente/cuerpo, hombre/mujer, naturaleza/cultura...es claro que el pensamiento occidental ha estado profundamente influido por las dicotomías: cuerpo/alma y naturaleza/cultura. 
En su texto, Las Tres Ecologías (1989) Guattari dice que es evidente que los contenidos de la subjetividad se han hecho cada vez más dependientes de una multitud de sistemas mecánicos, y este cambio de la subjetividad se acelera en el siglo XVIII, con el desequilibrio producido en; "las relaciones del ser humano con la herramienta."(Guattari, 1996; 15-18). Lo anterior modificó, gracias a la estructura del capital, las territorialidades que configuraban la subjetividad del individuo. Ese desequilibrio ha aumentado en la era que Guattari llama de la informatización planetaria, un nuevo ambiente en que la máquina se coloca bajo el control de la subjetividad: no una subjetividad humana reterritorializada, sino una nueva clase de subjetividad mecánica. El autor entiende por ecosofía una articulación ético-política entre los tres registros ecológicos, el del medio ambiente, el de las relaciones sociales y el de la subjetividad humana:

"Las formaciones políticas y las instancias ejecutivas se muestran totalmente incapaces de aprehender esta problemática (las medioambientales) en el conjunto de sus implicaciones, en general se limitan a abordar el campo de la contaminación industrial, pero exclusivamente desde una perspectiva tecnocrática, cuando en realidad solo una articulación ético-política -que yo llamo ecosofía- entre los tres registros eco lógicos, el del medio ambiente, el de las relaciones sociales y el de la subjetividad humana, sería susceptible de clarificar convenientemente estas cuestiones" (Guattari, 1996; 21-23).

Respecto a la ecosofía mental, Guattari nos dice:

"Se verá obligada a reinventar la relación del sujeto con el cuerpo, el fantasma, la finitud del tiempo, los “misterios" de la vida y de la muerte. Se verá obligada a buscar antídotos a la uniformización «mass-mediática» y telemática, al conformismo de las modas, a las manipulaciones de la opinión por la publicidad, los sondeos, etc.” (Guattari, 1996; 57) 
Lo anterior implica aceptar la tarea de construir los límites de la vida diaria en conexión parcial con los otros en comunicación con todas nuestras partes.

La corporeidad no comienza ni termina en la piel del sujeto, menos aún en el tiempo presente, donde asistimos a la emergencia de subjetivaciones sin sujetos o sujetos vacios, pero lo que no implica que estén vaciados de cuerpos. "Esto sugiere de entrada reconocer toda una serie de mutaciones culturales producidas por el impacto de la técnica en nuestra vida cotidiana.’'(Scolari, 2015; 38). Citando a Deleuze podemos decir que:

"El cuerpo sin órganos se opone al organismo, no al cuerpo, deshacer el organismo nunca ha sido matarse, sino abrir el cuerpo a conexiones que suponen todo un agenciamiento, circuitos, conjunciones, niveles y umbrales, pasos y distribuciones de intensidad, territorios y desterritorializaciones medidas a la manera de un agrimensor." (Deleuze, 200; 196).

La naturaleza es rehecha a través de la técnica y finalmente se convertirá en algo artificial, al tiempo que la cultura se convierte en algo natural. Entonces ¿La evolución humana depende de su interacción con la tecnología? Al respecto Dagognet va incluso más lejos y afirma que el carácter maleable de la naturaleza revela una invitación a lo artificial; "La naturaleza es un bricoleur ciego, una lógica primaria de combinaciones que produce infinidad de diferencias posibles.” (Dagognet, 2008; 34)

Al parecer, lo nuevo hay que imaginárselo como una interminable variación de lo ya existente. La innovación no opera con las cosas mismas, sino con las jerarquías culturales y los valores, o sea transmutar el valor de algo visto y conocido desde siempre. La visualidad hoy presenta una estetización de la realidad que es también una visualización de la imagen más completa de esa misma realidad. Si toda realidad ha devenido imagen entonces en la misma medida se torna más difícil conceptualizar una experiencia específica de la imagen que se distinguiera de otras formas de experiencias. Entonces el cuerpo, es capaz de liberarse de las representaciones y de los controles que produce. Al decir de Boris Groys; "Este cuerpo, donde todos los estados corporales se mezclan simultáneamente.” (Groys, 2008; 209) 
La corporalidad en la técnica se construye: hay nueva producción del cuerpo, una nueva forma de producción de cuerpos, la relación como espacio de construcción y entendimiento. O en palabras sencillas, hacer de otro modo. Este hacer de otro modo, nos ubica frente a variadas prótesis cuerpos e intenciones maquínicas siendo el cuerpo una entidad integral constituida por diversas dimensiones y complementarias entre sí. En su complementariedad explica su poder performativo. "El cuerpo es un locus en el ejercicio del poder." (Foucault, 2008; 153)

El cuerpo es el nodo en que convergen las diferentes estructuras de la sociedad por lo tanto viene siendo objeto de estudio de la experiencia humana y sus manifestaciones donde se sintetizan las relaciones de poder.

Décadas anteriores Claude Lévi-Strauss consideraba oportuno aportar con informaciones sobre la importancia de las tecnologías corporales para los procesos de intercambio y contactos culturales. "Por lo mismo no se puede negar que las tecnologías corporales dejan muchas huellas sobre los efectos del poder.” (Lévi-Strauss, 1971; 16-17)

\section{Conclusión:}

En el cuerpo ocurre la trazabilidad de mapas en todos sus territorios hasta los lugares más remotos. Vemos esa liminalidad entre un cuerpo y otro incrustado en la carne: cuerpos en transición o fronterizos, o de representación. Hoy no sólo hay una correlación entre el hombremáquina, como lo sería un humano usando una herramienta, sino que las nuevas prótesis han dado origen a una progresiva simbiosis entre el cuerpo y la máquina que se va abriendo paso a paso en el organismo. El planteamiento de esas alteraciones en la información de la especie humana apunta a la mutación de los cuerpos y subjetividades. La sociedad se adaptará de diferentes maneras y por lo tanto desconocidas. "La imagen del cuerpo como laboratorio de experiencias se ha convertido en una realidad omnipresente y volátil, difícil de localizar, sin límites fijos, que muta." (Mayayo, 2004; 85-125). Da la impresión de que el cuerpo, cuando se muestra, no lo hace sino para plantear una serie de interrogantes sobre la propia corporeidad. Sobre este momento, Scolari basándose en las reflexiones de Guattari dice que:

"Es evidente que los contenidos de la subjetividad se han hecho cada vez más dependientes de una multitud de sistemas mecánicos y este cambio de 
la subjetividad se acelera en el siglo XVIII con el desequilibrio producido en "las relaciones del ser humano con la herramienta" que modificó gracias a la estructura del capital, de las territorialidades que configuran la subjetividad del individuo. El cuerpo como territorialización-desterritorialización donde se alzan todos los discursos acerca del cuerpo." (Scolari, 2015; 32)

Esta metáfora de la mecánica del cuerpo es analizada por Foucault en Vigilar y Castigar, (1975) quien entiende que la racionalización del cuerpo como máquina es la forma del control de las fuerzas de trabajo que debe proporcionar el sujeto en las instituciones, es decir, que el sometimiento de los cuerpos a unas formas para la eficacia de las acciones emprendidas busca la docilidad de los sujetos. "Lo que se llama disciplina no es otra cosa que la forma general de dominación." (Foucault, 1998; 183).

La misma noción de lenguaje nos sirve para integrar los cambios materiales en la producción capitalista asociada a la información cuando palabras propias de los programas informáticos empiezan a vincularse con el lenguaje cotidiano. Nuevas formas de producción y un nuevo sensorium, marca la aparición de sociedades conectadas que se comportan y usan el lenguaje para transformarlo en algo nuevo.

Estas contradicciones pero al mismo tiempo estas posibilidades de construir un discurso alternativo, han sido iluminadas gracias al concepto de rizoma, (Deleuze y Guattari, 2000; 214). Útil para metaforizar la estructura formal de Internet cuanto para teorizar las potencialidades del modelo de discurso contemporáneo, un discurso cuyo potencial consiste en dejar espacios a las minorías, a la contrahegemonía, a luchas sociales y valores alternativos a los postulados que detentan el poder.

El sentido del cuerpo actual, situado aquí y ahora, incluso sin extensiones materiales de ningún tipo, trasciende los límites físicos. El reto está en enfrentarse a esta complejidad: aún queda mucho espacio para levantar otros muchos discursos dedicados a las cuestiones que no hemos podido desarrollar. Lo que sí es cierto es que la vida va sucediendo a nuestro alrededor a través de la pantalla, nuestros cuerpos que somatizan se vuelven otros. En este sentido podemos decir que el cuerpo es un potencial de transformación que no requiere de una separación con la materia, sino hacerla y tomarla como intensa y fluctuante, lo suficiente para poder decir que se ha 
Andrea Miranda

puesto en crisis su actual organización para inclinarse hacia otras, nuevas, formas de composición.

Hemos visto cómo el cuerpo ha sido modificado en su naturaleza, y este cambio tiene que ver también con los avances biotecnológicos. La tecnología se ha emparejado con la evolución del hombre hace un par de décadas y se encuentra en íntima relación con el cuerpo, que es también y simultáneamente un nuevo cuerpo. "Dando expresión a la realidad de los variables procesos de formación ya no de individuos sino que de singularidades." (Ruiz Stull, 2011; 133$135)$.

Es por esto que solo lo que involuciona evoluciona, ya que la potencia de transformación no se entiende desde la adición de partes sobre partes para formar una suma total cerrada sobre sí misma; sino al contrario, requiere de un primer deshacerse de la organización para así transformarse.

\section{Bibliografía}

Abril Curto Gonzalo. (2010) El cuarto bios. Estudios sobre comunicación e información. Madrid. Ed. Complutense.

Adorno, Theodor; Horkheimer Max. (1994) M. Dialéctica de la Ilustración. Ed. Trotta. Madrid. Aguilar García, Teresa. (2008) Ontología cyborg. Barcelona: Gedisa.

Arendt, Hannah. (1993) La condición humana. Barcelona, Paidós.

Bataille, Georges. (1996) El ojo Pineal; Precedido de El año Solar; Y Sacrificios. Valencia. Ed. Pre-Textos.

Dagognet, Jerome. (2008) Introducción a la medicina experimental. Ed. Flammarion, París.

Deleuze, Gilles. (2001) Spinoza: Filosofía Práctica. Ed. Tusquets

Foucault. Michel. (2008) Historia de la Sexualidad. Vol I. La voluntad de saber. Buenos Aires. Ed. Siglo XXI.

Foucault, Michel. (2005) Historia de la Sexualidad. Vol II. El uso de los placeres. Ed Siglo XXI. Buenos Aires. Argentina.

Foucault, Michel. (1998) Vigilar y Castigar. Nacimiento de la prisión. Ed. Siglo XXI, México.

Guattari, Félix. (1996) Las Tres Ecologías. Valencia. Ed. Pre-Textos. 
Andrea Miranda

Groys, Boris. (2008) Política de la Inmortalidad. Cuatro conversaciones con Thomas Knoefel. Buenos Aires. Katz Editores

Lévi-Strauss Claude. (1979) Introducción a la obra de Marcel Mauss en Sociología y antropología. Madrid. Ed. Tecnos.

Mayayo, Patricia (2004) "La reinvención del cuerpo" en Ramirez, J.A. y Carillo, J. (eds.), Tendencias del arte, arte de tendencias a principios del siglo XXI, Ensayos Arte. Madrid. Ed. Cátedra.

Marcuse. Herbert. (2003) Eros y Civilización. Ed. Ariel. Barcelona.

Merleau-Ponty, Maurice. (1999) Fenomenología de la Percepción. Barcelona. Ed.Península Merleau-Ponty, Maurice. (2010) Lo visible y lo invisible. Buenos Aires: Ed. Ed. Nueva Visión Merleau-Ponty, Maurice. (2002) El mundo de la Percepción. Buenos Aires: Ed. FCE

Miñambres, Carmelo (Comp). (2002) “Pensando, el cuerpo, pensando desde el cuerpo” ed. Popular Libros. Albacete.

Nietzsche, Friedrich. (2000) Schopenhauer como Educador. Madrid. Ed. Biblioteca Nueva.

Ruiz Stull, Miguel. (2011) "La fórmula del cuerpo sin órganos una aproximación bergsoniana a su enunciación”. Trans/Form/Ação vol.34 no.1 Marília 2011, https://www.scielo.br/pdf/trans/v34n1/a08v34n1.pdf 131-135

Scolari, Carlos. (2015) Ecología de los medios: Entornos, Evoluciones, Representaciones. Barcelona. Ed. Gedisa. 